\title{
EXAMPLES FOR THE NONLOCALLY CONVEX THREE SPACE PROBLEM
}

\author{
M. RIBE
}

\begin{abstract}
A simple way to obtain certain examples of locally bounded spaces $E$ of the following kind is described: $E$ is nonlocally convex but contains a locally convex subspace $K$ such that $E / K$ is locally convex.
\end{abstract}

The purpose of this note is to give a quite simple construction which proves the following:

THEOREM 1 (Independently proved by Kalton [5]). There is a separated locally bounded space $E$ with an uncomplemented one-dimensional subspace $L$ such that $E / L$ is isomorphic to $l^{1}$.

THEOREM 2. Indeed, there is an uncountable class of such spaces $E$ of which no one can be mapped into another by a continuous linear mapping which does not annihilate the uncomplemented line.

THEOREM 3A. There is a locally bounded space $E$ with a subspace $K$ such that $K$ is isomorphic to $l^{\infty}$ and $E / K$ to $l^{1}$, and such that for any infinite-dimensional subspace $E_{1} \subset E \backslash K$, the subspace $E_{1}+K$ fails to be locally convex.

THEOREM 3B. Let $\Lambda$ be a set of the cardinality of the continuum. There is a locally bounded space $E$ with a subspace $K$ such that $K$ is isomorphic to $l^{\infty}(\Lambda)$ and $E / K$ to $l^{1}$, and such that for any infinite-dimensional subspace $E_{1} \subset$ $E \backslash K$, the subspace $E_{1}+K$ fails to be separated by its dual.

Kalton [5] also proves that in Theorem $1, l^{1}$ cannot be replaced by $l^{p}$ for any $p \neq 1(0<p<\infty)$. For some further recent related results, see Dierolf [1], Kalton and Peck [6], and Roberts [9]; cf. also Ribe [7].

We are thus dealing with the so-called three space problem, which is of current interest also in the pure Banach space setting; some results have been given by Enflo, Lindenstrauss, and Pisier [2]. In the desire to grasp the nature of this problem it is apparently well motivated to study cases where transparent solutions are possible.

Received by the editors November 9, 1977 and, in revised form, April 14, 1978.

AMS (MOS) subject classifications (1970). Primary 46A10; Secondary 46B05.

Key words and phrases. Locally bounded space, quotient space, three space problem, dualseparation. 
The author is grateful to Professor N. J. Kalton and to the referee for their very valuable remarks.

\section{The construction.}

Definition. A nonnegative functional $\|\cdot\|$ on a linear space is a quasinorm if for some real constant $k \geqslant 1$, all $x$ and $y$ in the space satisfy

(i) $\|x+y\| \leqslant k(\|x\|+\|y\|)$.

(ii) $\|s x\|=|s|\|x\|$ for all scalars $s$.

(iii) $\|x\|=0$ implies $x=0$.

A quasi-norm defines a separated locally bounded topology on the space, with 0 -neighbourhood base $\{\{x \mid\|x\|<\varepsilon\} \mid \varepsilon>0\}$. The smallest possible $k$ value is sometimes called the modulus of concavity of $\|\cdot\|$.

LEMMA 1. Let $F$ and $G$ be quasi-normed spaces. Let $\phi: G \rightarrow F$ be a mapping which for all $x, y$ in $G$ fulfills the conditions:

(i) $\|\phi(x)+\phi(y)-\phi(x+y)\|_{F} \leqslant c\left(\|x\|_{G}+\|y\|_{G}\right)-\|x+y\|_{G}$ for some constant $c \geqslant 1$.

(ii) $\phi(s x)=s \phi(x)$ for all scalars $s$.

Then the linear space $F \times G$ can be made into a quasi-normed space $E$ so that for $(x, y)$ in $E$ we have:

$1^{\circ} .\|(x, 0)\|_{E}=\|x\|_{F}$.

$2^{\circ} .\|(x, y)\|_{E} \geqslant\|(\phi(y), y)\|_{E}=\|y\|_{G}$.

So in particular $E / F$ and $G$ are isomorphic.

(Remark. If $k$ is the modulus of concavity, we can actually achieve $3^{\circ} . k_{E} \leqslant \max \left(k_{F}, c\right)$.)

Proof. We just have to put

$$
\|(x, y)\|_{E}=\|x-\phi(y)\|_{F}+\|y\|_{G}
$$

A straightforward computation, using the assumptions for $\phi$, and the properties of $\|\cdot\|_{F}$ and $\|\cdot\|_{G}$ as quasi-norms, shows that this is a quasi-norm. It obviously has the properties $1^{\circ}$ and $2^{\circ}$. (Further, it has property $3^{\circ}$.)

Note that the conditions on the mapping $\phi$ are a sort of "control of its nonlinearity". For the construction of such a mapping we shall use a simple inequality.

LEMMA 2. For all real numbers $s, t$ we have

$$
|s \log | s|+t \log | t|-(s+t) \log | s+t|| \leqslant 2(|s|+|t|) .
$$

(We put $0 \log 0=0$.)

Proof. Case I: $t \geqslant s>0$. Then the left-hand side is equal to

$$
\begin{aligned}
s \log ((s+t) / s)+t \log ((s+t) / t) \\
\quad<t \cdot 2|(s / 2 t) \log (s / 2 t)|+t \log 2<2 t<2(s+t) .
\end{aligned}
$$

Case II: $t<-s<0$. Reduced to Case I by the substitution $s^{\prime}=s$, $t^{\prime}=-s-t$. 
Let $\|\cdot\|_{1}$ be the usual norm on $l_{R}^{1}$, and let $l_{0}^{1}$ be the subspace of all finitely nonzero sequences. Applying Lemma 2 we get:

LEMMA 3. Define a functional $\varphi: l_{0}^{1} \rightarrow \mathbf{R}$ by

$$
\varphi(x)=\sum_{i} x_{i} \log \left|x_{i}\right|-\left(\sum_{i} x_{i}\right) \log \left|\sum_{i} x_{i}\right|,
$$

where $x=\left(x_{i}\right)_{i>1}$. Then for $x, y$ in $l_{0}^{1}$ we have:

$$
\begin{aligned}
& 1^{\circ} \cdot|\varphi(x)+\varphi(y)-\varphi(x+y)| \leqslant 4\left(\|x\|_{1}+\|y\|_{1}\right) . \\
& 2^{\circ} \cdot \varphi(s x)=s \varphi(x) \text { for all reals } s .
\end{aligned}
$$

Proof of Theorem 1. Consider the case with real scalars. By Lemma 3 we can apply Lemma 1 with $F=\mathbf{R}, G=l_{0}^{1}$, and $\phi=\varphi$ (taking $c=5$ ). Replacing the space $E$ so obtained with its completion we get the desired $E$.

Namely, for all integers $n \geqslant 2$ and $k, 1 \leqslant k \leqslant n$, define points $y^{(n, k)}$ in $l_{0}^{1}$ with the coordinates

$$
\begin{aligned}
y_{i}^{(n, k)} & =1 / \log n \quad \text { for } i=k, \\
& =-\frac{1}{n-1}\left(\frac{1}{\log n}\right) \text { for other } i, 1 \leqslant i \leqslant n, \\
& =0 \text { for all other } i .
\end{aligned}
$$

Then we get

$$
\left\|y^{(n, k)}\right\|_{1}=2 / \log n
$$

and

$$
\varphi\left(y^{(n, k)}\right)=1+o(1)
$$

as $n \rightarrow \infty$. Since

$$
(1,0)=(1 / n) \sum_{k}\left(1, y^{(n, k)}\right)
$$

for each $n$, the statements $1^{\circ}$ and $2^{\circ}$ of Lemma 1 imply that the point $(1,0)$ lies in the convex hull of every 0 -neighbourhood in $E$. Hence $(1,0)$ cannot be separated from 0 by any continuous linear form, and hence the line $\operatorname{lin}\{(1,0)\}$ is uncomplemented in $E$.

(Remark. By replacing $\varphi$ with $\varepsilon \varphi$ in this proof, with $\varepsilon>0$ small, we can make the modulus of concavity of $E$ arbitrarily close to 1.)

Let us close the section with some remarks on dual-separation in nonconvex spaces. Hahn-Banach's theorem easily gives:

Proposition. The space $E$ and the one-dimensional subspace $L$ in Theorem 1 have this property: The weak closure of any closed subspace $E_{1}$ in $E$ is equal to $E_{1}+L$.

So in particular $E$ is an $F$-space which is not locally convex but yet contains no proper closed weakly dense (abbreviated PCWD) subspace. (Roberts [9] obtained yet another such space.) It is perhaps somewhat 
surprising that there is such a simple and natural example of a space with this property. For this is in striking contrast to the following sort of converse to Hahn-Banach's theorem, very recently proved by Kalton [4] (improving Corollary 5.3 of Kalton [3]): If a separable $F$-space with separating dual has no PCWD subspace, then it is locally convex.

Further, consider an algebraic supplement $K$ to $L$ in $E$. Then $K$ is a nonlocally convex metrizable space which has the HBEP; i.e., the HahnBanach Extension Property, meaning that the dual separates points from closed subspaces. (Namely, if $K_{1}$ is any relatively closed subspace in $K$, its closure in $E$ either contains $L$ or is equal to $K_{1}$; in both cases, $K_{1}$ must be relatively weakly closed in $K$, by the Proposition.) But by Corollary $\mathrm{E}$ of Ribe [8], any $l^{p}$-sum (where $0<p \leqslant \infty$ ) of infinitely many copies of $K$ fails to have the HBEP. So the HBEP is not generally preserved under completion, and not generally preserved under formation of $l^{p}$-sums for any $p$.

Many very different isomorphy types. To prove Theorem 2, let us first state an elementary observation. On a given quasi-normed space, define functionals $r_{k}(x)$, for all $k \geqslant 1$, as the infimum of those numbers $r>0$ for which $x$ lies in the convex hull of some subset of cardinality $k$ in the open $r$-neighbourhood of zero. Now, for any continuous linear mapping $f$ between two quasi-normed spaces satisfying $\|f(x)\| \leqslant C\|x\|$ (say), we have $r_{k}(f(x)$ ) $\leqslant C r_{k}(x)$ for all $k$.

Proof of TheOrem 2. Let $G_{1}, G_{2}, \ldots$ be subspaces in $l^{1}$ with $\operatorname{dim} G_{i}=i$, spanned by disjoint sets of basis vectors; let $P_{i}$ be the projection onto $G_{i}$. Now, for any given bounded number sequence $\alpha_{1}, \alpha_{2}, \ldots$, let $E\left(\alpha_{1}, \alpha_{2}, \ldots\right)$ denote that space $E$ which is obtained as in the proof of Theorem 1, but with $\varphi$ replaced by $\Sigma_{i} \alpha_{i} \varphi P_{i}$. Then let $R_{k}\left(\alpha_{1}, \alpha_{2}, \ldots\right)$ be the number $r_{k}(e)$, where $e$ is the point $(1,0)$ in $E\left(\alpha_{1}, \alpha_{2}, \ldots\right)$.

We shall recursively define a nonnegative sequence $\varepsilon_{1}, \varepsilon_{2}, \ldots$, with $\varepsilon_{i}=0$ except for $i=n(k)(k \geqslant 1)$, say. Put $\varepsilon_{1}=1$. Now suppose that $\varepsilon_{i}$ has been found for $i \leqslant n=n(k)$; we want to define $n(k+1)$ and $\varepsilon_{n(k+1)}$. First, there is a number $\delta>0$ such that

$$
\begin{aligned}
R_{j}\left(\sigma_{1} \varepsilon_{1}, \ldots, \sigma_{n} \varepsilon_{n}, \gamma_{n+1}, \gamma_{n+2}, \ldots\right) \\
\\
>\left(1-2^{-k}\right) R_{j}\left(\sigma_{1} \varepsilon_{1}, \ldots, \sigma_{n} \varepsilon_{n}, 0,0, \ldots\right)
\end{aligned}
$$

when $j \leqslant k$, for all choices of $\left\{\sigma_{i}\right\}$ with $\sigma_{i}=0$ or 1 , and for all choices of $\gamma_{i}$ with $\left|\gamma_{i}\right| \leqslant \delta$. Then we put $\varepsilon_{n(k+1)}=\delta$, where $n(k+1)=m>n$ is taken so large that

$$
\begin{aligned}
& R_{m}\left(\sigma_{1} \varepsilon_{1}, \ldots, \sigma_{m} \varepsilon_{m}, 0,0, \ldots\right) \\
&<(1 / k) R_{m}\left(\sigma_{1} \varepsilon_{1}, \ldots, \sigma_{n} \varepsilon_{n}, 0,0, \ldots\right)
\end{aligned}
$$

for all $\left\{\sigma_{i}\right\}$ with $\sigma_{i}=0$ or 1 , and $\sigma_{m}=1$. For by the argument in the proof of Theorem 1 we can find such an $m$.

In view of the remark before the proof we can now find uncountably many 
spaces $E\left(\sigma_{1} \varepsilon_{1}, \sigma_{2} \varepsilon_{2}, \ldots\right)$, with $\sigma_{i}=0$ or 1 , which fulfill our claim.

\section{Strictly singular quotient mappings.}

Proof of Theorem 3A. Let $\left\{S_{i}\right\}$ be an enumeration of the set of all finite subsets in the set of the usual basis vectors in $l^{1}$, and let $P_{i}$ be the projection onto the space spanned by $S_{i}$. In analogy to the proof of Theorem 1, we define $E$ as the completion of the space $E$ obtained when we apply Lemma 1 with $F=l^{\infty}, G=l_{0}^{1}$, and $\phi=\left(\varphi P_{i}\right)_{i}$.

Let $X$ be any infinite-dimensional subspace in $l^{1}$. Then for any integer $n \geqslant 1$ and any number $\varepsilon>0$ there are unit vectors $x^{(1)}, \ldots, x^{(n)}$ in $l^{1}$, which are supported by disjoint finite sets of basis vectors and which have $\operatorname{dist}\left(x^{(i)}, X\right)<\varepsilon$. Choose an $m$ such that each of the vectors $P_{m}\left(x^{(i)}\right)$ has length at least $1 / 2$ and is such that its coordinates do not have different signs. We can then apply the argument in the proof of Theorem 1, with the role of the basis vectors played by those vectors $P_{m}\left(x^{(i)}\right)$ which have nonnegative coordinates, or those which have nonpositive coordinates. Since $n$ and $\varepsilon$ are arbitrary, this argument implies that the inverse image of $X$ in $E$ is not locally convex.

Theorem 3B is proved in the same way, but with $\left\{S_{i}\right\}$ replaced by the set of all subsets of the basis.

Specifically, the quotient mappings of Theorems $3 \mathrm{~A}$ and $3 \mathrm{~B}$ are strictly singular, i.e., each of their restrictions to infinite-dimensional subspaces fails to be an isomorphism.

\section{REFERENCES}

1. S. Dierolf, Über Vererbbarkeitseigenschaften in topologischen Vektorräumen, Dissertation, Munich 1974.

2. P. Enflo, J. Lindenstrauss and G. Pisier, On the "three space problem", Math. Scand. 36 (1975), 199-210.

3. N. J. Kalton, Basic sequences in F-spaces and their applications, Proc. Edinburgh Math. Soc. 19 (1974), 151-167.

4. Quotients of $F$-spaces (to appear).

5.

6. N. J. Kalton and N. T. Peck, Quotients of $L^{P}(0,1)$ for $0<p<1$, Notices Amer. Math. Soc. 24 (1977), p. A-115; (to appear).

7. M. Ribe, On the separation properties of the duals of general topological vector spaces, Ark. Mat. 9 (1971), 279-302.

8. Necessary convexity conditions for the Hahn-Banach theorem in metrizable spaces, Pacific J. Math. 44 (1973), 715-732.

9. J. Roberts, $A$ nonlocally convex $F$-space with the Hahn-Banach approximation property, Banach Spaces of Analytic Functions, Lecture Notes in Math., vol. 604, Springer-Verlag, Berlin, 1977, pp. 76-81.

Lostigen 1, S-17171 SOLNa, Sweden 